\title{
SHARP STRICHARTZ ESTIMATES FOR THE SCHRÖDINGER EQUATION ON THE SPHERE
}

\author{
DUVÁN CARDONA AND LILIANA ESQUIVEL
}

\begin{abstract}
In this contribution we investigate the Schrördinger equation associated to the Laplacian on the sphere in the form of sharp Strichartz estimates. We will provided simple proofs for our main theorems using purely the $L^{2} \rightarrow L^{p}$ spectral estimates for the operator norm of the spectral projections (associated to the spherical harmonics) proved in [8]. A sharp index of regularity is established for the initial data in spheres of arbitrary dimension $d \geqslant 2$.
\end{abstract}

\section{INTRODUCTION}

In this work we provide sharp Strichartz estimates for the Schrödinger equation for the Laplacian on the sphere $\mathbb{S}^{d}, \Delta_{\mathbb{S}^{d}}$, which is given by

$$
(\mathrm{PVI}): \begin{cases}i u_{t}+\Delta_{\mathbb{S}^{d}} u=0, & (t, x) \in \mathbb{T} \times \mathbb{S}^{d}, \\ u(0, x)=f(x), & x \in \mathbb{S}^{d},\end{cases}
$$

where $\mathbb{T} \cong[0,2 \pi), 0 \sim 2 \pi$, denotes the one dimensional torus. The fractal nature of the solutions for (1.1) has been discussed, e.g. in Taylor [15] and references therein. To illustrate our results let us recall, that (1.1) is an analogy of the standard Schrödinger equation on $\mathbb{R}^{n}$,

$$
i u_{t}(t, x)+\Delta_{\mathbb{R}^{n}} u(t, x)=0,
$$

with initial data $u(0, \cdot)=f$. The solution for (1.2), satifies the following Strichartz estimate

$$
\left\|e^{i t \Delta_{\mathbb{R}^{n}}} f\right\|_{L^{q}\left[(0, \infty), L_{x}^{p}\left(\mathbb{R}^{d}\right)\right]} \leqslant C\|f\|_{L^{2}\left(\mathbb{R}^{n}\right)},
$$

which holds, if and only if, $1 / q=n / 2(1 / 2-1 / p)$, for $n=1$ and $2 \leqslant p \leqslant \infty$, $n=2$ and $2 \leqslant p<\infty$, and, $2 \leqslant p<\frac{2 n}{n-2}$ if $n \geqslant 3$. This fundamental result proved by Keel and Tao [7] is the source of inspiration for several extensions of (1.2) to general compact and non-compact manifolds.

The key point for obtaining (1.3), is the following estimate

$$
\left\|e^{i t \Delta_{\mathbb{R}^{n}}}\right\|_{L^{1}\left(\mathbb{R}^{n}\right) \rightarrow L^{\infty}\left(\mathbb{R}^{n}\right)} \lesssim_{n} C|t|^{-\frac{n}{2}}
$$

which expresses the dispersive property of the linear Schrödinger equation on $\mathbb{R}^{n}$. If $\mathbb{R}^{n}$ is replaced by a compact manifold $M$, the analogue of the dispersion estimate (1.4) fails globally in time, as the example of constant solutions shows

2010 Mathematics Subject Classification. Primary: 35Q40, Secondary: 42B35, 42C10, $35 \mathrm{~K} 15$.

The first author was supported by the FWO Odysseus 1 grant G.0H94.18N: Analysis and Partial Differential Equations of Professor Michael Ruzhansky. 
(see Burq, Gerard and Tzvetkov [4] for details). It is less obvious, but still true, that it also fails locally in time (see the discussion in the beginning of Section 3 of [4]). For the case of the $d$-dimensional torus, $M=\mathbb{T}^{d}$, Bourgain $[1,2,3]$ proposed a different approach to Strichartz estimates by using the Fourier series representation of the Schrödinger group,

$$
u(t, x) \equiv e^{i t \Delta_{\mathbb{T}^{d}}} f(x):=\sum_{k \in \mathbb{Z}^{d}} e^{i 2 \pi\left(t|k|^{2}+k \cdot x\right)} \hat{f}(k) .
$$

Notice that this representation shows that the solution is also periodic in time. By computing explicitly

$$
\|u\|_{L^{4}\left(\mathbb{T} \times \mathbb{T}^{d}\right)}^{4}=\left\|u^{2}\right\|_{L^{2}\left(\mathbb{T} \times \mathbb{T}^{d}\right)}^{2},
$$

Bourgain obtains similar Strichartz estimates as in the fundamental paper due to Keel and Tao [7].

In general, on a compact manifold without boundary $M$, it was proved by Burq, Gerard and Tzvetkov [4] the following estimate

$$
\left\|e^{i t \Delta_{M}} f\right\|_{L^{q}\left[[0, T], L_{x}^{p}(M)\right]} \lesssim_{T, p, q, s}\|f\|_{W^{s}(M)}, \quad s \geqslant \frac{1}{q}
$$

for $1 / q=\operatorname{dim}(M) / 2(1 / 2-1 / p), q \geqslant 2$, and $p<\infty$. As in our case, Taylor in [15] considered the case of spheres $M=\mathbb{S}^{d}$, where the author obtained sharp $L^{p}$-estimates on the solution operator at those times which are rational multiples of $\pi$. This idea that the profile of linear dispersive equations depend on the algebraic properties of time have been further exploited in the papers of KapitanskiRodniaski [6], Taylor [15] and Rodnianski [14] where the fractal nature for the solution of the Schrödinger equation on spheres and torus were considered.

Our main goal is to study (1.1), by using the $L^{2} \rightarrow L^{p}$ bounds for the spectral resolution of the Laplacian $\Delta_{\mathbb{S} d}$. More precisely, we deal with the problem of establishing an analogy of (1.2) in the case of the Laplacian on the sphere $\mathbb{S}^{d}$. Our main results are, the following Theorem 1.1, and Theorem 1.2, where the Schrödinger equation with potential (4.1), (this means, associated to $-\Delta_{\mathbb{S}^{d}}+$ $V(x, t)$, for a suitable potential $V(x, t))$ is considered.

Theorem 1.1. Let $d \geqslant 2$ and $p, q$ satisfy $2 \leqslant p \leqslant \infty$, and $2 \leqslant q<\infty$. Then the following Strichartz estimate

$$
\left\|e^{i t \Delta_{\mathbb{S}^{d}}} f(z)\right\|_{L_{z}^{p}\left[\mathbb{S}^{d}, L_{t}^{q}(\mathbb{T})\right]} \lesssim_{p, q, s}\|f\|_{W^{s}\left(\mathbb{S}^{d}\right)}{ }^{1}
$$

holds true for $s \geqslant \varkappa_{p, q}$, where

$$
\varkappa_{p, q}:=\left\{\begin{array}{lr}
\frac{d-1}{2}\left(\frac{1}{2}-\frac{1}{p}\right)+\left(\frac{1}{2}-\frac{1}{q}\right), & 2 \leqslant p \leqslant \frac{2(d+1)}{d-1}, \\
d\left(\frac{1}{2}-\frac{1}{p}\right)-\frac{1}{q}, & p>\frac{2(d+1)}{d-1} .
\end{array}\right.
$$

\footnotetext{
${ }^{1}$ Observe that under the symmetric compromise $p=q,\|u(t, z)\|_{L_{z}^{p}\left[\mathbb{S}^{d}, L_{t}^{q}(\mathbb{T})\right]}=$ $\|u(t, z)\|_{L^{p}\left(\mathbb{S}^{d} \times \mathbb{T}\right)}$, and for $p=4,\|u\|_{L^{4}\left(\mathbb{S}^{d} \times \mathbb{T}\right)}^{4}=\left\|u^{2}\right\|_{L^{2}\left(\mathbb{S}^{d} \times \mathbb{T}\right)}^{2}$. We use the notation $A \lesssim B$ to indicate $A \leqslant c B$, where $c>0$ does not depend on fundamental quantities.
} 
For $q=2$, and $s<\varkappa_{p, 2}$, the estimate in (1.7) does not hold for any non-trivial initial data $f \in L^{2}\left(\mathbb{S}^{d}\right)$. So, in this case, the regularity order $\varkappa_{p, 2}$ is sharp in any dimension $d$, in the sense that (1.7) does not hold for all $s<\varkappa_{p, 2}$.

By keeping the notation $\varkappa_{p, 2}$ in Theorem 1.1, we consider the case of Schrödinger equation associated with the Hamiltonian

$$
\mathbb{H}:=-\Delta_{\mathbb{S}^{d}}+V(x, t) .
$$

Indeed, we prove:

Theorem 1.2. Let us consider the following Schrödinger equation with potential

$$
\left(\mathrm{PVI}^{\prime}\right): \begin{cases}i u_{t}=\left(-\Delta_{\mathbb{S}^{d}}+V(x, t)\right) u, & (t, x) \in \mathbb{T} \times \mathbb{S}^{d}, \\ u(0, x)=f(x), & x \in \mathbb{S}^{d}\end{cases}
$$

where $\|V\|_{L_{x}^{q}\left(\mathbb{S}^{d}, L_{t}^{\infty}(\mathbb{T})\right)}$ is small enough. Let us assume that $\frac{1}{q}+\frac{1}{p / 2}=1$. Then, there exists a unique solution $u \in C\left(\mathbb{T}, W_{x}^{s}\left(\mathbb{S}^{d}\right)\right) \cap L_{x}^{p}\left(\mathbb{S}^{d}, L_{t}^{2}(\mathbb{T})\right)$ for $(1.8)$, provided that $s \geqslant \varkappa_{p, 2}$

This paper is organised as follows. In Section 2 we present some basic properties about the spectral decomposition of the Laplacian and we introduce some auxiliar (Triebel-Lizorkin) spaces, that will be useful in our analysis. In Section 3 we prove our Theorem 1.1. Finally, in Section 4 we consider the Schrödinger equation with potential. We finish this introduction by discussing briefly our main results.

Remark 1.3. For $M=\mathbb{S}^{d}$, let us observe that (1.6) is in general a better estimate (in the case when $p>2(d+1) /(d-1)$ ) that the obtained in Theorem 1.1 in the case, where,

$$
1 / q=d / 2(1 / 2-1 / p),^{2}
$$

but, both estimates agree for $2 \leqslant p \leqslant 2(d+1) /(d-1)$. However, the main point in Theorem 1.1 is the sharpness of the index $\varkappa_{p, 2}$ in any dimension, and that the estimate that we obtain is in general independent of the known restriction $1 / q=\operatorname{dim}(M) / 2(1 / 2-1 / p)$, between the dimension $d$, and the pair $(p, q)$. Several sharp results on the sphere are know only for slow dimensions $d$, (see e.g. Taylor [15]), or for $p=q=4$ (see Burq, Gerard and Tzvetkov [4]), but the sharp Sobolev regularity order $\varkappa_{p, 2}$ is to our knowledge, a new result in this context and also in the context of Theorem 1.2 for the Hamiltonian $\mathbb{H}$.

Remark 1.4. We will discuss some implications of our results to general manifolds by following a similar analysis as in Ruzhansky and Turunen [16, page 578]. Under the existence of a $C^{\infty}$-diffeomorphism $i: \mathbb{S}^{d} \rightarrow M$, where $M$ is a Riemannian manifold, (these kind of diffeomorphisms are known for $d=3$, with $M$ being a connected a simply connected manifold in view of the Perelman proof $[9,10,11$, 12] for the Poincaré conjecture [13] and in higher dimensions), if $X_{1}, \cdots, X_{d}$ is an orthonormal basis for the tangent bundle $T \mathbb{S}^{d}$, in such a way that

$$
\Delta_{\mathbb{S}^{d}}:=-\sum_{1 \leqslant j \leqslant d} X_{j}^{2}
$$

\footnotetext{
${ }^{2}$ By following the usual nomenclature, such pair $(p, q)$ is called admissible.
} 
let us denote by

$$
\Delta_{M}:=-\sum_{1 \leqslant j \leqslant d} Y_{j}^{2}
$$

the second order differential operator induced by $i$. This means that, $Y_{j}:=i_{*}\left(X_{j}\right)$, is the vector field induced by the differential of $i$, which is a diffeomorphism between tangent bundles, $i_{*}: T \mathbb{S}^{d} \rightarrow T M$. Theorem 1.1 implies the estimate

$$
\left\|e^{i t \Delta_{M}} u_{0}\right\|_{L_{z}^{p}\left[M, L_{t}^{q}(\mathbb{T})\right]} \leqslant C\left\|u_{0}\right\|_{W^{s}(M)}
$$

for $s \geqslant \varkappa_{p, q}$, with $\varkappa_{p, 2}$ being sharp, and also Theorem 1.2, implies the existence of a unique $u \in C\left(\mathbb{T}, W_{x}^{s}(M)\right) \cap L_{x}^{p}\left(M, L_{t}^{2}(\mathbb{T})\right)$ satisfying the differential problem

$$
(\mathrm{PVI} "): \begin{cases}i u_{t}=\left(-\Delta_{M}+V(x, t)\right) u=0, & (t, x) \in \mathbb{T} \times M, \\ u(0, x)=u_{0}(x), & x \in M,\end{cases}
$$

for a potential $V$ with $\|V\|_{L_{x}^{q}\left(M, L_{t}^{\infty}(\mathbb{T})\right)}$ small enough.

\section{PRELIMINARIES}

Now we present some basics about the spectral decomposition of the Laplacian on the sphere, for this we follow Schoen and Yau [17]. Let $d \geqslant 1, d \in \mathbb{N}$. Let

$$
\mathbb{S}^{d}:=\left\{x=\left(x_{0}, x_{1}, \cdots, x_{d}\right) \in \mathbb{R}^{d+1}:\left|x_{0}\right|^{2}+\left|x_{1}\right|^{2}+\cdots+\left|x_{d}\right|^{2}=1\right\},
$$

be the $d$-dimensional unit sphere contained in $\mathbb{R}^{d+1}$ and $\mathcal{H}_{n}^{d}$ be the space of spherical harmonics of degree $n$. There is a standard metric $g$ on $\mathbb{S}^{d}$, induced by the standard metric on $\mathbb{R}^{d+1}$, and conrrespondly, a Laplace operator $\Delta_{\mathbb{S}}^{d}$ which can be defined, initially on $C^{\infty}\left(\mathbb{S}^{d}\right)$ as follows. For $f \in C^{\infty}\left(\mathbb{S}^{d}\right)$, let $\tilde{f}(x):=f(x /|x|)$, then

$$
\Delta_{\mathbb{S}^{d}} f:=\left.\Delta_{\mathbb{R}^{d+1}} \tilde{f}\right|_{\mathbb{S}^{d}}
$$

where, by following the usual nomenclature,

$$
\Delta_{\mathbb{R}^{d+1}}:=-\partial_{x_{0}}^{2}-\partial_{x_{1}}^{2}-\cdots-\partial_{x_{d}}^{2}
$$

is the standard positive Laplacian on $\mathbb{R}^{d+1}$. It can be shown that for $r=|x|$, one can write

$$
\Delta_{\mathbb{R}^{d+1}} \tilde{f}(x)=r^{-2}\left(\Delta_{\mathbb{S}^{d}} f\right) .
$$

In polar coordinates $r=|x|, \omega=x / r$,

$$
\Delta_{\mathbb{S}^{d}}=r^{2} \Delta_{\mathbb{R}^{d+1}}-r^{2} \partial_{r}^{2}-d r \partial_{r}
$$

It is well known, from the Fourier analysis associated to $\Delta_{\mathbb{S}^{d}}$, the spectral decomposition

$$
L^{2}\left(\mathbb{S}^{d}\right)=\bigoplus_{n=0}^{\infty} \mathcal{H}_{n}^{d}, \operatorname{dim}\left(\mathcal{H}_{n}^{d}\right) \sim n^{d-1}
$$


where $L^{2}\left(\mathbb{S}^{d}\right) \equiv L^{2}\left(\mathbb{S}^{d}, d x\right)$ is the usual $L^{2}$-space ${ }^{3}$ associated to the surface measure on the sphere, $d x:=d \sigma_{\mathbb{S}^{d}}(x)$, and that, $\mathcal{H}_{n}^{d}$ is the eigenspace of the LaplaceBeltrami operator $\Delta_{\mathbb{S}^{d}}$ associated with the eigenvalue $\lambda_{n}=n(n+d-1)$. Let $H_{n}^{d}$ be the spectral projection to the subspace $\mathcal{H}_{n}^{d}$ for every $n \in \mathbb{N}_{0}$. Then, for every $f \in L^{2}\left(\mathbb{S}^{d}\right)$ we can write

$$
f=\sum_{n=0}^{\infty} H_{n}^{d} f
$$

where the series converges in the $L^{2}\left(\mathbb{S}^{d}\right)$-norm. In particular, the spectral theorem for $\Delta_{\mathbb{S}^{d}}$ implies

$$
m\left(\Delta_{\mathbb{S}^{d}}\right)=\sum_{n=0}^{\infty} m\left(\lambda_{n}\right) H_{n}^{d} f
$$

for every continuous function $m$ on $\mathbb{R}$, where the domain of $m\left(\Delta_{\mathbb{S}^{d}}\right)$ is given by

$$
\operatorname{Dom}\left(m\left(\Delta_{\mathbb{S}^{d}}\right)\right):=\left\{f \in L^{2}\left(\mathbb{S}^{d}\right): \sum_{n=0}^{\infty}\left|m\left(\lambda_{n}\right)\right|^{2}\left\|H_{n}^{d} f\right\|_{L^{2}\left(\mathbb{S}^{d}\right)}^{2}<\infty\right\} .
$$

By observing that, for $m(\lambda):=e^{ \pm i t \lambda}, \operatorname{Dom}\left(m\left(\Delta_{\mathbb{S}^{d}}\right)\right)=L^{2}\left(\mathbb{S}^{d}\right)$, the Schrödinger propagator $e^{i t \Delta_{\mathbb{S}^{d}}}: L^{2}\left(\mathbb{S}^{d}\right) \rightarrow L^{2}\left(\mathbb{S}^{d}\right)$ is given by

$$
e^{i t \Delta_{\mathbb{S}^{d}}} f:=\sum_{n=0}^{\infty} e^{i \lambda_{n} t} H_{n}^{d} f
$$

and for $f \in C^{\infty}\left(\mathbb{S}^{d}\right)$ the solution to (1.1) is given by

$$
u(t, x)=\sum_{n=0}^{\infty} e^{i \lambda_{n} t} H_{n}^{d} f(x), \quad(t, x) \in \mathbb{T} \times \mathbb{S}^{d} .
$$

In this paper we want to estimate the mixed norms $L_{x}^{p}\left(L_{t}^{q}\right)$ of solutions to Schrödinger equations by using the following version of Triebel-Lizorkin associated to the family of spectral projections of the Laplacian $\Delta_{\mathbb{S}^{d}}$.

Definition 2.1. Let us consider $0<p \leqslant \infty, r \in \mathbb{R}$ and $0<q \leqslant \infty$. The TriebelLizorkin space associated to the family of projections $H_{n}^{d}, n \in \mathbb{N}_{0}$, and to the parameters $p, q$ and $r$ is defined by those complex functions $f$ satisfying

$$
\|f\|_{F_{p, q}^{r}\left(\mathbb{R}^{n}\right)} \equiv\|f\|_{F_{p, q}^{r}\left(\mathbb{R}^{n},\left(H_{n}^{d}\right)_{n \in \mathbb{N}}\right)}:=\left\|\left(\sum_{n=0}^{\infty} n^{r q}\left|H_{n}^{d} f\right|^{q}\right)^{\frac{1}{q}}\right\|_{L^{p}\left(\mathbb{S}^{d}\right)}<\infty .
$$

The definition considered above differs from those arising with dyadic decompositions on compact homogeneous manifolds (see e.g. Nursultanov, Ruzhansky and Tikhonov [5]). Indeed, when working with dyadic decompositions one recover

\footnotetext{
${ }^{3}$ As usually, defined by those measurable functions on $\mathbb{S}^{d}$, such that $\|f\|_{L^{2}\left(\mathbb{S}^{d}\right)}:=$ $\left(\int_{\mathbb{S}^{d}}|f(\omega)|^{2} d \omega\right)^{\frac{1}{2}}<\infty$. In general, on a measure space $(X, d x)$, we use $\|f\|_{L^{p}(X)}:=$ $\left(\int_{X}|f(x)|^{p} d x\right)^{\frac{1}{p}}$, for all $1 \leqslant p<\infty$.
} 
the Littlewood-Paley theorem, about the equivalence of certain dyadic TriebelLizorkin spaces with the Lebesgue spaces $L^{p}\left(\mathbb{S}^{d}\right)$, which with the current definition we do not necessarily have. The following are natural embedding properties of such spaces. Sobolev spaces $W^{s, p}$ in $L^{p}\left(\mathbb{S}^{d}\right)$-spaces and associated to $\Delta_{\mathbb{S}^{d}}$, can be defined by the norm

$$
\|f\|_{W^{s, p}} \equiv\left\|\Delta_{\mathbb{S}^{d}}^{s} f\right\|_{L^{p}}:=\left(\int_{\mathbb{S}^{d}}\left|\Delta_{\mathbb{S}^{d}}^{s} f(x)\right|^{p} d x\right)^{\frac{1}{p}} 4 .
$$

We will use the notation $W^{s}:=W^{s, 2}$ for the Sobolev space modelled on $L^{2}\left(\mathbb{S}^{d}\right)$. Indeed, we have

$$
\|u\|_{W^{s}\left(\mathbb{S}^{d}\right)}=\left(\sum_{\ell=0}^{\infty} \ell^{s}\left\|H_{\ell}^{d} u\right\|_{L^{2}\left(\mathbb{S}^{d}\right)}\right)^{\frac{1}{2}}{ }^{5} .
$$

The following properties can be deduced by following a similar analysis as in $[5]$.

- $F_{p, q_{1}}^{r+\varepsilon} \hookrightarrow F_{p, q_{1}}^{r} \hookrightarrow F_{p, q_{2}}^{r} \hookrightarrow F_{p, \infty}^{r}, \varepsilon>0,0<p \leqslant \infty, 0<q_{1} \leqslant q_{2} \leqslant \infty$.

- $F_{p, q_{1}}^{r+\varepsilon} \hookrightarrow F_{p, q_{2}}^{r}, \varepsilon>0,0<p \leqslant \infty, 1 \leqslant q_{2}<q_{1}<\infty$.

- $F_{2,2}^{0}=L^{2}$ and consequently, for every $s \in \mathbb{R}, W^{s}=F_{2,2}^{s}$.

It is a very special fact, how at rational multiples of $\pi$, the propagator $e^{i t \Delta_{\mathbb{S}^{d}}}$ behaves on Sobolev spaces. Indeed, let us remark that (see Proposition 2.1 of Taylor [15]) for all $1<p<\infty$, and all $s \in \mathbb{R}$,

$$
e^{2 \pi i\left(\frac{m}{2 k}\right) \Delta_{\mathbb{S} d}}: W^{s, p}\left(\mathbb{S}^{d}\right) \rightarrow W^{s-(d-1)\left|\frac{1}{2}-\frac{1}{p}\right|, p}\left(\mathbb{S}^{d}\right),
$$

extends to a bounded operator. For the sharpness of this estimate we refer the reader to Taylor [15, page 148$]$.

\section{Proof of Theorem 1.1}

In this section we will prove one of our main results. We will see that information about the Strichtaz estimates for the Schrödinger propagator, is encoded in the $L^{2} \rightarrow L^{p}$-operator norm of the projections $H_{n}^{d}$, and this will essential in order to give a short proof for Theorem 1.1.

The space $L_{\text {fin }}^{2}\left(\mathbb{S}^{d}\right)$ consists of those finite linear combinations of elements in the sub-spaces $\mathcal{H}_{n}^{d}$.

Lemma 3.1. Let us consider $f \in F_{p, 2}^{0}\left(\mathbb{S}^{d}\right)$, then for all $1 \leqslant p \leqslant \infty$ we have

$$
\|u(t, z)\|_{L_{z}^{p}\left[\mathbb{S}^{d}, L_{t}^{2}(\mathbb{T})\right]}=\sqrt{2 \pi}\|f\|_{F_{p, 2}^{0}\left(\mathbb{S}^{d}\right)} .
$$

\footnotetext{
${ }^{4}$ Here, $W^{s, p}:=\operatorname{Dom}\left(\Delta_{\mathbb{S}^{d}}^{s}\right)$ is considered as the completion of $C^{\infty}\left(\mathbb{S}^{d}\right)$ with respect to the norm defined in (2.3).

${ }^{5}$ Where we use $=$ because $\ell \sim \lambda_{\ell}:=[\ell(\ell+d-1)]^{\frac{1}{2}}$ for $\ell \rightarrow \infty$.
} 
Proof. Let us consider $f \in F_{p, 2}^{0}\left(\mathbb{S}^{d}\right)$. By a standard argument of density we only need to assume $f \in L_{\text {fin }}^{2}\left(\mathbb{S}^{d}\right)$. The solution $u(t, z)$ for $(1.1)$ is given by

$$
u(t, z)=\sum_{n=0}^{\infty} e^{i t \lambda_{n}} H_{n}^{d} f(z) .
$$

Let us note that the previous sums runs over a finite number of $n^{\prime} s$ because $f \in L_{\text {fin }}^{2}\left(\mathbb{S}^{d}\right)$. So, we have,

$$
\|u(t, z)\|_{L_{t}^{2}(\mathbb{T})}^{2}=\int_{0}^{2 \pi} u(t, z) \overline{u(t, z)} d t=\int_{0}^{2 \pi} \sum_{n, n^{\prime}} e^{i t\left(\lambda_{n}-\lambda_{n^{\prime}}\right)} H_{n}^{d} f(z) \overline{H_{n^{\prime}}^{d} f(z)} d t .
$$

Observe that if $n \neq n^{\prime}$, then

$$
\lambda_{n}=n(n+d-1) \neq n^{\prime}\left(n^{\prime}+d-1\right)=\lambda_{n^{\prime}}
$$

and consequently, from the $L^{2}$-orthogonality of the trigonometric polynomials we get,

$$
\begin{aligned}
\|u(t, z)\|_{L_{t}^{2}(\mathbb{T})}^{2} & =\int_{0}^{2 \pi} u(t, z) \overline{u(t, z)} d t=\sum_{n, n^{\prime}} \int_{0}^{2 \pi} e^{i t\left(\lambda_{n}-\lambda_{n^{\prime}}\right)} H_{n}^{d} f(z) \overline{H_{n^{\prime}}^{d} f(z)} d t \\
& =\sum_{n=0}^{\infty} 2 \pi \cdot\left|H_{n}^{d} f(z)\right|^{2},
\end{aligned}
$$

where we have changed summation with integration because $f \in L_{\text {fin }}^{2}\left(\mathbb{S}^{d}\right)$. Thus, we conclude the following fact

$$
\|u(t, z)\|_{L_{t}^{2}(\mathbb{T})}=\left(\sum_{n=0}^{\infty} 2 \pi \cdot\left|H_{n}^{d} f(z)\right|^{2}\right)^{\frac{1}{2}}, f \in L_{\mathrm{fin}}^{2}\left(\mathbb{S}^{d}\right) .
$$

Finally,

as claimed.

$$
\|u(t, z)\|_{L_{2}\left(\mathbb{S}^{d}, L_{t}^{2}(\mathbb{T})\right)}=\sqrt{2 \pi}\|f\|_{F_{p, 2}^{0}\left(\mathbb{S}^{d}\right)},
$$

The following lemma, allows us to compare the $L_{z}^{p}\left(L_{t}^{q}\right)$-mixed norms of the solution $u(t, z)$ and some Triebel-Lizorkin norms of the initial data.

Lemma 3.2. Let $p, q$ and $s_{q}$ be such that $0<p \leqslant \infty, 2 \leqslant q<\infty$ and $s_{q}:=\frac{1}{2}-\frac{1}{q}$. Then

$$
C_{p}^{\prime}\|f\|_{F_{p, 2}^{0}} \leqslant\|u(t, z)\|_{L_{z}^{p}\left(\mathbb{S}^{d}, L_{t}^{q}(\mathbb{T})\right)} \leqslant C_{p, s}\|f\|_{F_{p, 2}^{s}}
$$

holds true for every $s \geqslant s_{q}$.

Proof. Let us consider, by a density argument, the initial data $f \in L_{f}^{2}\left(\mathbb{R}^{n}\right)$. In order to estimate the norm $\|u(t, z)\|_{L_{z}^{p}\left[\mathbb{S}^{d}, L_{t}^{q}(\mathbb{T})\right]}$ we can use the Wainger Sobolev embedding Theorem:

$$
\left\|\sum_{n \in \mathbb{Z}, n \neq 0}|n|^{-\alpha} \hat{F}(n) e^{i n t}\right\|_{L^{q}(\mathbb{T})} \leqslant C\|F\|_{L^{r}(\mathbb{T})}, \quad \alpha:=\frac{1}{r}-\frac{1}{q} .
$$


For $s_{q}:=\frac{1}{2}-\frac{1}{q}$ we have

$$
\begin{aligned}
\|u(t, z)\|_{L^{q}(\mathbb{T})} & =\left\|\sum_{n=0}^{\infty} e^{i t \lambda_{n}} H_{n}^{d} f(z)\right\|_{L^{q}(\mathbb{T})} \\
& \leqslant C\left\|\sum_{n=0}^{\infty} n^{s_{q}} e^{i t \lambda_{n}} H_{n}^{d} f(z)\right\|_{L^{2}(\mathbb{T})} \\
& =C\left\|\sum_{n=0}^{\infty} e^{i t \lambda_{n}} H_{n}^{d}\left[\mathcal{L}^{s_{q}} f(z)\right]\right\|_{L^{2}(\mathbb{T})} \\
& =C\left(\sum_{n=0}^{\infty} \mid H_{n}^{d}\left[\left.\mathcal{L}^{s_{q}} f(z)\right|^{2}\right)^{\frac{1}{2}}\right. \\
& :=T^{\prime}\left(\mathcal{L}^{s_{q}} f\right)(z),
\end{aligned}
$$

where we have denoted

$$
\mathcal{L}^{s_{q}}:=\sum_{n=0}^{\infty} n^{s_{q}} H_{n}^{d}
$$

So, we have

$$
\begin{aligned}
\|u(t, z)\|_{L_{z}^{p}\left[\mathbb{S}^{d}, L_{t}^{q}(\mathbb{T})\right]} & \leqslant C\left\|T^{\prime}\left(H^{s_{q}} f\right)\right\|_{L^{p}(\mathbb{S} d)} \\
& =C\left\|\mathcal{L}^{s_{q}} f\right\|_{F_{p, 2}^{0}\left(\mathbb{S}^{d}\right)} \\
& =C\|f\|_{F_{p, 2}^{s_{q}}\left(\mathbb{S}^{d}\right)} .
\end{aligned}
$$

We end the proof by taking into account the embedding $F_{p, 2}^{s} \hookrightarrow F_{p, 2}^{s_{q}}$ for every $s \geqslant s_{q}$, and the following estimate for all $2 \leqslant q<\infty$,

$$
\|f\|_{F_{p, 2}^{0}} \lesssim\left\|T^{\prime} f\right\|_{L^{p}}=C\|u(t, z)\|_{L_{z}^{p}\left[\mathbb{S}^{d}, L_{t}^{2}(\mathbb{T})\right]} \lesssim\|u(t, z)\|_{L_{z}^{p}\left[\mathbb{S}^{d}, L_{t}^{q}(\mathbb{T})\right]}
$$

The following lemma, about the $L^{2} \rightarrow L^{p}$-operator norm of the spectral projection for the Laplacian on the sphere, is a consequence of the main results in Kwon and Lee [8].

Lemma 3.3. Let $n \in \mathbb{N}$, and let $2 \leqslant p \leqslant \infty$. Let us consider the orthogonal projection $H_{n}^{d}: L^{2}\left(\mathbb{S}^{d}\right) \rightarrow \mathcal{H}_{n}^{d}, n \in \mathbb{N}$. Then we have

$$
\left\|H_{n}^{d} f\right\|_{L^{p}\left(\mathbb{S}^{d}\right)} \leqslant C n^{\varkappa_{p}}\|f\|_{L^{2}\left(\mathbb{S}^{d}\right)},
$$

where

$$
\varkappa_{p}:=\frac{d-1}{2}\left(\frac{1}{2}-\frac{1}{p}\right), \quad \text { if, } 2 \leqslant p \leqslant \frac{2(d+1)}{d-1}
$$

and

$$
\varkappa_{p}:=d\left(\frac{1}{2}-\frac{1}{p}\right)-\frac{1}{2}, \quad \text { if, } p>\frac{2(d+1)}{d-1} .
$$

The exponent $\varkappa_{p}$ is sharp, in the sense that there is not $f \in L^{2}\left(\mathbb{S}^{d}\right), f \neq 0$, such that

$$
\left\|H_{n}^{d} f\right\|_{L^{p}\left(\mathbb{S}^{d}\right)} \leqslant C^{\prime} n^{s}\|f\|_{L^{2}\left(\mathbb{S}^{d}\right)}
$$


for all $s<\varkappa_{p}$.

Now, with the analysis developed above and by using Theorem 3.3 we will provide a short proof for the main result of this section.

Proof of Theorem 1.1. We observe that from Lemma 3.2,

$$
C_{p, s}^{\prime}\|f\|_{F_{p, 2}^{0}} \leqslant\|u(t, z)\|_{L_{z}^{p}\left(\mathbb{S}^{d}, L_{t}^{q}(\mathbb{T})\right)} \leqslant C_{p, s}\|f\|_{F_{p, 2}^{s}}
$$

so, we only need to estimate the $F_{p, 2}^{s}$-norm of the initial data $f$, by showing that $\|f\|_{F_{p, 2}^{s}} \lesssim\|f\|_{W^{s}}$, for every $s \geqslant \varkappa_{p, q}$. Moreover, by the embedding $W^{s} \hookrightarrow W^{\varkappa_{p, q}}$ for every $s \geqslant \varkappa_{p, q}$ we only need to check the previous estimate when $s=\varkappa_{p, q}$. By the condition $2 \leqslant p<\infty$, together with the Minkowski integral inequality and Theorem 3.3, we give

$$
\begin{aligned}
\|f\|_{F_{p, 2}^{s q}} & =\left\|\left(\sum_{n=0}^{\infty} n^{2 s_{q}}\left|H_{n}^{d} f(z)\right|^{2}\right)^{\frac{1}{2}}\right\|_{L^{p}} \\
& \leqslant\left(\sum_{n=0}^{\infty} n^{2 s_{q}}\left\|H_{n}^{d} f\right\|_{L^{p}}^{2}\right)^{\frac{1}{2}} \\
& \lesssim\left(\sum_{n=0}^{\infty} n^{2\left(s_{q}+\varkappa_{p}\right)}\left\|H_{n}^{d} f\right\|_{L^{2}}^{2}\right)^{\frac{1}{2}} \\
& =\|f\|_{W^{\varkappa_{p}, q},}
\end{aligned}
$$

where we have used that $\varkappa_{p, q}=s_{q}+\varkappa_{p}$. Let us note that the previous estimates are valid for $p=\infty$. The sharpness of the result for $q=2$ can be proved by choosing $f=H_{n}^{d} g$ for some arbitrary, but non-trivial function $g \in L^{2}\left(\mathbb{S}^{d}\right)$. In this case, $\varkappa_{p, q}=\varkappa_{p}$ because $s_{q}=0$, and we have

$$
\begin{aligned}
\|u(t, z)\|_{L_{z}^{p}\left[\mathbb{S}^{d}, L_{t}^{2}(\mathbb{T})\right]} & =\left\|H_{n}^{d} g\right\|_{L^{p}} \leqslant n^{\varkappa_{p}}\left\|H_{n}^{d} g\right\|_{L^{2}} \\
& =\|g\|_{W^{\varkappa_{p}}} .
\end{aligned}
$$

The sharpness of this inequality is consequence of the sharpness for the exponent $\varkappa_{p}$ in Lemma 3.3. Indeed, an improvement of the previous estimate would yield improved estimates for the spectral projection operator $H_{n}^{d}$, which is not possible. Thus, we finish the proof.

\section{The Schrödinger Equation With POTEntial.}

In this section we study the problem,

$$
\left(\mathrm{PVI}^{\prime}\right): \begin{cases}i u_{t}=\left(-\Delta_{\mathbb{S}^{d}}+V(x, t)\right) u, & (t, x) \in \mathbb{T} \times \mathbb{S}^{d}, \\ u(0, x)=f(x), & x \in \mathbb{S}^{d}\end{cases}
$$

for a suitable potential $V$. Our starting point is the following lemma. 
Lemma 4.1. The Schrödinger propagator satisfies

$$
\left\|\int_{0}^{t} e^{i(t-\tau) \Delta_{\mathbb{S}^{d}}}(G(\cdot, \tau))(x) d \tau\right\|_{L_{x}^{p}\left(\mathbb{S}^{d}, L_{t}^{2}(\mathbb{T})\right)} \leqslant C\|G\|_{L_{x}^{p^{\prime}}\left(\mathbb{S}^{d}, L_{t}^{2}(\mathbb{T})\right)}
$$

Proof. Using the duality argument, we have

$$
\begin{aligned}
& \left\|\int_{0}^{t} e^{i(t-\tau) \Delta_{\mathbb{S}^{d}}}(G(\cdot, \tau))(x) d \tau \mid\right\|_{L_{x}^{p}\left(\mathbb{S}^{d}, L_{t}^{2}(\mathbb{T})\right)} \\
& =\sup \left\{\left|\int_{\mathbb{S}^{d}} \int_{0}^{2 \pi} \int_{0}^{t} e^{i(t-\tau) \Delta_{\mathbb{S}^{d}}}(G(\cdot, \tau))(x) d \tau h(x, t) d t d x\right|:\|h\|_{L_{x}^{p^{\prime}}\left(\mathbb{S}^{d}, L_{t}^{2}(\mathbb{T})\right)}=1\right\} .
\end{aligned}
$$

Now, since $\Delta_{\mathbb{S}^{d}}$ is a positive operator, this implies $e^{i t \Delta_{\mathbb{S}^{d}}}$ is unitary, this by various applications of Fubini's theorem we have

$$
\begin{aligned}
\int_{\mathbb{S}^{d}} \int_{0}^{2 \pi} \int_{0}^{t} e^{i(t-\tau) \Delta_{\mathbb{S}^{d}}(G(\cdot, \tau))}(x) d \tau h(x, t) d t d x \\
=\int_{0}^{2 \pi} \int_{0}^{t} \int_{\mathbb{S}^{d}}^{t} e^{i(t-\tau) \Delta_{\mathbb{S}^{d}}}(G(x, \tau))(x) h(x, t) d x d \tau d t \\
=\int_{\mathbb{S}^{d}}\left(\int_{0}^{t} e^{-i \tau \Delta_{\mathbb{S}^{d}}} G(x, \tau) d \tau\right)\left(\int_{0}^{2 \pi} e^{\left.i t \Delta_{\mathbb{S}^{d}} h(x, t) d t\right) d x,}\right.
\end{aligned}
$$

via Hlder's inequality we have

$$
\begin{aligned}
& \left|\int_{\mathbb{S}^{d}} \int_{0}^{2 \pi} \int_{0}^{t} e^{i(t-\tau) \Delta_{\mathbb{S} d}}(G(\cdot, \tau))(x) d \tau h(x, t) d t d x\right| \\
& \leqslant\left\|\int_{0}^{2 \pi} e^{-i \tau \Delta_{\mathbb{S}^{d}}} G(x, \tau) d \tau\right\|_{L^{2}\left(\mathbb{S}^{d}\right)}\left\|\int_{0}^{2 \pi} e^{i t \Delta_{\mathbb{S}^{d}}} h(x, t) d t\right\|_{L^{2}\left(\mathbb{S}^{d}\right)} .
\end{aligned}
$$

Since

$$
\left\|\int_{0}^{2 \pi} e^{-i \tau \Delta_{\mathbb{S}^{d}}} G(x, t) d t\right\|_{L^{2}\left(\mathbb{S}^{d}\right)} \leqslant C\|G\|_{L_{x}^{p^{\prime}}\left(\mathbb{S}^{d}, L_{t}^{2}(\mathbb{T})\right)}
$$

from (4.3) and (4.4) we have (4.2)

We define the Banach space

$$
X:=C\left(\mathbb{T}, W_{x}^{s}\left(\mathbb{S}^{d}\right)\right) \cap L_{x}^{p}\left(\mathbb{S}^{d}, L_{t}^{2}(\mathbb{T})\right)
$$

via the norm

$$
\|u\|_{X}:=\sup _{t \in \mathbb{T}}\|u(t, \cdot)\|_{L_{z}^{2}}+\|u\|_{L_{x}^{p}\left(\mathbb{S}^{d}, L_{t}^{2}(\mathbb{T})\right)}
$$


Now we prove the main result of this section.

Proof of Theorem 1.2. In view of the Duhamel principle, the solution for (PVI') can be written as

$$
u(t, z)=e^{i t \Delta_{\mathbb{S} d}} f(x)-\int_{0}^{t} e^{i(t-\tau) \Delta_{\mathbb{S} d}}(V(\cdot, \tau) u(\cdot))(x) d \tau .
$$

We use the standard contraction mapping argument, we need to prove the nonlinear map

$$
\Phi w:=e^{i t \Delta_{\mathbb{S}^{d}}} f(x)-\int_{0}^{t} e^{i(t-\tau) \Delta_{\mathbb{S}^{d}}}(V(\cdot, \tau) w(\cdot))(x) d \tau .
$$

defines a contraction map on $X$. From the unitary group properties and the estimation given in Theorem 1.1 there exists $C_{0}>1$ such that

$$
\left\|e^{i t \Delta_{\mathbb{S}^{d}}} f(x)\right\|_{X} \leqslant C_{0}\|f\|_{W^{s}\left(\mathbb{S}^{d}\right)} .
$$

Now we estimate the integral part in (4.8). Using (4.2) and the estimation in Theorem 1.1 for $q=2$, we also have

$$
\| \int_{0}^{t} e^{i(t-\tau) \Delta_{\mathbb{S}^{d}}}\left(V(\tau, \dot{)} w(\cdot))(x) d \tau\left\|_{X} \leqslant\left(C_{0}+C_{0}^{2}\right)\right\| V w \|_{X}\right.
$$

Note, $\|V w\|_{L_{x}^{p^{\prime}\left(\mathbb{S}^{d}, L_{t}^{2}(\mathbb{T})\right)}}, \leqslant\|V\|_{L_{x}^{q}\left(\mathbb{S}^{d}, L_{t}^{\infty}(\mathbb{T})\right)}\|w\|_{L_{x}^{p}\left(\mathbb{S}^{d}, L_{t}^{2}(\mathbb{T})\right)}$, as long as $\frac{1}{q}+\frac{2}{p}=1$, therefore

$$
\| \int_{0}^{t} e^{i(t-\tau) \Delta_{\mathbb{S} d}}\left(V(\tau, \dot{)} w(\cdot))(x) d \tau\left\|_{X} \leqslant\left(C_{0}+C_{0}^{2}\right)\right\| V\left\|_{L_{x}^{q}\left(\mathbb{S}^{d}, L_{t}^{\infty}(\mathbb{T})\right)}\right\| w \|_{X} .\right.
$$

Hence

$$
\|\Phi w\| \leqslant C_{0}\|f\|_{W^{s}\left(\mathbb{S}^{d}\right)}+\left(C_{0}+C_{0}^{2}\right)\|V\|_{L_{x}^{q}\left(\mathbb{S}^{d}, L_{t}^{\infty}(\mathbb{T})\right)}\|w\|_{X}
$$

So, if we take $\|V\|_{L_{x}^{q}\left(\mathbb{S}^{d}, L_{t}^{\infty}(\mathbb{T})\right)}$, being small enough, such that

$$
\left(C_{0}+C_{0}^{2}\right)\|V\|_{L_{x}^{q}\left(\mathbb{S}^{d}, L_{t}^{\infty}(\mathbb{T})\right)} \leqslant \frac{1}{2}
$$

we observe, $\Phi$ maps $B=\left\{w \in X:\|w\|_{X} \leqslant 2\left(C_{0}+1\right)\|f\|_{W^{s}\left(\mathbb{S}^{d}\right)}\right\}$ into itself. Now, if $w, v \in B$, the same argument shows that

$$
\|\Phi(w)-\Phi(v)\|_{X} \leqslant \frac{1}{2}\|w-v\|_{X}
$$

Thus, by the contraction mapping principle, there exists a unique solution to (4.1). Thus, we finish the proof. 


\section{REFERENCES}

1. Bourgain, Jean. "Fourier transform restriction phenomena for certain lattice subsets and applications to nonlinear evolution equations." Geometric \& Functional Analysis GAFA 3.3 (1993): 209-262.

2. Bourgain, Jean. "Exponential sums and nonlinear Schrdinger equations." Geometric \& Functional Analysis GAFA 3.2 (1993): 157-178.

3. Bourgain Jean. "Global solutions of nonlinear Schrodinger equations." Vol. 46. American Mathematical Soc., 1999.

4. Burq Nicolas, Patrick Grard, and Nikolay Tzvetkov. "The Schrdinger equation on a compact manifold: Strichartz estimates and applications." Journes quations aux drives partielles (2001): 1-18.

5. Nursultanov Erlan, Michael Ruzhansky, and Sergey Tikhonov. "Nikolskii inequality and Besov, Triebel-Lizorkin, Wiener and Beurling spaces on compact homogeneous manifolds." Ann. Sc. Norm. Super. Pisa Cl. Sci. (5) 16 (2016), no. 3, 981-1017.

6. Kapitanski Lev, Rodnianski Igor. "Does a Quantum Particle Know the Time?" Emerging Applications of Number Theory. Volumes in Mathematics and its Applications, vol. 109, Springer Verlag, New York, 1999, pp 355-371

7. Keel, Markus, and Terence Tao. "Endpoint strichartz estimates." American Journal of Mathematics 120.5 (1998): 955-980.

8. Kwon, Yehyun, and Sanghyuk Lee. "Sharp $L^{p}-L^{q}$ estimates for the spherical harmonic projection (Harmonic Analysis and Nonlinear Partial Differential Equations)." RIMS Kokyuroku Bessatsu 70 (2018): 33-58.

9. Perelman, Grisha. "Spaces with curvature bounded below." Proceedings of the International Congress of Mathematicians. Birkhuser, Basel, 1995.

10. Perelman, Grisha. "The entropy formula for the Ricci flow and its geometric applications." arXiv preprint math/0211159 (2002).

11. Perelman, Grisha. "Ricci flow with surgery on three-manifolds." arXiv preprint math/0303109 (2003).

12. Perelman, Grisha. "Finite extinction time for the solutions to the Ricci flow on certain three-manifolds, preprint, 2003." arXiv preprint math.DG/0307245 16

13. Poincar, Henry. H. "Cinquime complment lanalysis situs." Rendiconti del Circolo Matematico di Palermo (1884-1940) 18.1 (1904): 45-110.

14. Rodnianski, Igor. "Fractal solutions of the Schrodinger equation." Contemporary Mathematics 255 (2000): 181-188.

15. Taylor, Michael. "The Schrödinger equation on spheres." Pacific journal of mathematics 209.1 (2003): 145-155.

16. Ruzhansky, M. Turunen, V. Pseudo-differential Operators and Symmetries: Background Analysis and Advanced Topics Birkhäuser-Verlag, Basel, (2010)

17. Schoen, R., and S. T. Yau. "Conference Proceedings and Lecture Notes in Geometry and Topology." Lectures on Differential Geometry (1994).

DuvÁn CARdona SÁNChez:

Department of Mathematics: Analysis, Logic and Discrete Mathematics

Ghent University, Belgium

E-mail address duvanc306@gmail.com, duvan.cardonasanchez@ugent.be

LILIANA ESQUIVEL:

Gran Sasso Science Institute, CP 67100

L' Aquila, Italia.

E-mail address liliana.esquivel@gssi.it 